University of Wollongong

Research Online

Faculty of Engineering and Information

Faculty of Engineering and Information

Sciences - Papers: Part A

Sciences

$1-1-2006$

\title{
Direction-of-arrival estimation in the presence of unknown nonuniform
} noise fields

\author{
Yuntao Wu \\ Chinese Academy of Sciences \\ Chaohuan Hou \\ Chinese Academy Of Sciences \\ Guisheng liao \\ Xidian University \\ Qinghua Guo \\ City University of Hong Kong, qguo@uow.edu.au
}

Follow this and additional works at: https://ro.uow.edu.au/eispapers

Part of the Engineering Commons, and the Science and Technology Studies Commons

Research Online is the open access institutional repository for the University of Wollongong. For further information contact the UOW Library: research-pubs@uow.edu.au 


\title{
Direction-of-arrival estimation in the presence of unknown nonuniform noise fields
}

\author{
Abstract \\ A new method for estimating directions-of-arrival (DOA) of multiple spatial narrowband signals in the \\ presence of spatially nonuniform independent sensor noise with unknown covariance is presented. An \\ estimate of the colored noise-covariance matrix is given first. The received data for parameter estimation \\ is then prewhitened using the estimated noise covariance, hence, overcoming the highly biased \\ estimates. Furthermore, the performance improvement of standard MUSIC method is confirmed by \\ computer simulations. (c) 2006 IEEE.
}

\section{Keywords}

presence, unknown, nonuniform, noise, estimation, fields, direction, arrival

\section{Disciplines}

Engineering | Science and Technology Studies

\section{Publication Details}

Wu, Y., Hou, C., liao, G. \& Guo, Q. (2006). Direction-of-arrival estimation in the presence of unknown nonuniform noise fields. IEEE Journal of Oceanic Engineering, 31 (2), 504-510. 


\title{
Regular Issue Peer-Reviewed Technical Communication
}

\author{
Direction-of-Arrival Estimation in the Presence \\ of Unknown Nonuniform Noise Fields
}

\author{
Yuntao Wu, Chaohuan Hou, Guisheng Liao, and Qinghua Guo
}

\begin{abstract}
A new method for estimating directions-of-arrival (DOA) of multiple spatial narrowband signals in the presence of spatially nonuniform independent sensor noise with unknown covariance is presented. An estimate of the colored noise-covariance matrix is given first. The received data for parameter estimation is then prewhitened using the estimated noise covariance, hence, overcoming the highly biased estimates. Furthermore, the performance improvement of standard MUSIC method is confirmed by computer simulations.
\end{abstract}

Index Terms-Array signal processing, direction-of-arrival (DOA) estimation, nonuniform noise.

\section{INTRODUCTION}

D IRECTION-OF-ARRIVAL (DOA) estimation is an important research problem in array signal processing. As is well known, many high-resolution methods like MUSIC, ESPRIT, etc., can provide excellent asymptotic performance under the assumption of white Gaussian noise. According to the assumption, sensor noises are considered to be a zero-mean Gaussian process with covariance matrix $\sigma^{2} \mathbf{I}$, where $\mathbf{I}$ is an identity matrix. However, all conventional methods for DOA estimation are sensitive to the noise model [2] and the performance of DOA estimation degrades severely when the assumption is not met (i.e., in colored noise) [3]. In recent years, the problem of DOA estimation in colored noise was extensively addressed and many algorithms were also presented by using partial prior knowledge or parameterization of the signal or noise covariance (see [1]-[9]). In several real applications (for example, sparse array is used), the general colored noise assumption can be simplified by assuming the sensor noise to become spatially white [7]-[9]. In this case, the noise-covariance matrix of array can be represented by a diagonal matrix, but the sensor noise variances are no longer identical to each other (i.e., nonuniform independent sensor with hardware nonideality in receiving channels as well as noise). Such a noise model becomes relevant in situations

for sparse arrays with prevailing external noise (for example, reverberation noise in sonar or external seismic noise) [8]. This practical relevance of the nonuniform noise model is verified by real seismic array data processing in [8].

To the problem of DOA estimation in the presence of spatially nonuniform independent sensor noise, a class of so-called covariance difference techniques was first proposed in [6] and [7]. More recently, a modified maximum-likelihood (ML) DOA estimation method [8] has been also derived and its implementation was based on an iterative procedure which includes a step-wise concentration of the log-likelihood function with respect to the signal and noise nuisance parameters. However, it requires a highly nonlinear multiple-dimension search and is computationally inefficient, while it needs to have an appropriate initialized values for faster convergence.

The nonuniform noise variances may be estimated by collecting signal-free data from the sensor array. However, in a real application system, the noise parameters may be time-varying, and a signal-free environment is not always available. This appears to be a strong motivation to use fully important $a$ priori knowledge of the particular noise case and propose a method for estimation of the relevant parameters of the noise model. In this paper, an estimate of noise covariance in the presence of unknown nonuniform independent sensor noise is given first and, hence, some available high-resolution methods can be straightforwardly applied using the prewhitened array data. Simulation results show the performance of the MUSIC method for DOA estimation, which is greatly improved using the modified data.

\section{SIGNAL MODEL}

Let an array of $N$ sensors receive $P$ narrowband signals impinging from the sources with unknown DOAs $\left\{\theta_{1}, \ldots, \theta_{P}\right\}$. The sensor array outputs can be expressed as

$$
\mathbf{x}(t)=\mathbf{A}(\theta) \mathbf{S}(t)+\mathbf{N}(t)
$$

where

Manuscript received April 4, 2005; revised September 13, 2005; accepted February 2, 2006. This work is supported by National Natural Science Foundation of China under Grants 60472101 and 60472097. Associate Editor: D. Abraham.

Y. Wu and C. Hou are with the Integrated Digital System Laboratory, Institute of Acoustics, Chinese Academy of Sciences, Beijing 100080, China (e-mail: ytwu@sina.com).

G. Liao is with the National Key Laboratory for Radar Signal Processing, Xidian University, Xian 710071, China.

Q. Guo is with the Department of Electronic Engineering, City University of Hong Kong, Kowloon, Hong Kong.

Digital Object Identifier 10.1109/JOE.2006.875270

$$
\begin{aligned}
\mathbf{x}(t) & =\left[x_{1}(t), \ldots, x_{N}(t)\right]^{T} \text { array data vector; } \\
\mathbf{A}(\theta) & =\left[\mathbf{a}\left(\theta_{1}\right), \mathbf{a}\left(\theta_{2}\right), \ldots, \mathbf{a}\left(\theta_{P}\right)\right] \text { array manifold matrix; } \\
\mathbf{S}(t) & =\left[s_{1}(t), \ldots, s_{P}(t)\right]^{T} \text { source waveform vector; } \\
\mathbf{N}(t) & =\left[n_{1}(t), \ldots, n_{N}(t)\right]^{T} \text { sensor noise vector; } \\
\mathbf{a}\left(\theta_{i}\right) & =\text { steering vector; } \\
(\cdot)^{T} & =\text { transposition of a matrix. }
\end{aligned}
$$


From (1), the array covariance matrix is given by

$$
\begin{aligned}
\mathbf{R}_{\mathbf{x x}} & =E\left[\mathbf{x}(t) \mathbf{x}^{H}(t)\right] \\
& =\mathbf{A R}_{s s} \mathbf{A}^{H}+\mathbf{Q}
\end{aligned}
$$

where $(\cdot)^{H}$ denotes the Hermitian transpose and $\mathbf{R}_{s s}$ is the source covariance matrix. The sensor noise is assumed to be a zero-mean spatially and temporally white Gaussian process with the unknown diagonal covariance matrix (see [6]-[8]); therefore, we have the following:

$$
\mathbf{Q}=E\left\{\mathbf{N}(t) \mathbf{N}^{H}(t)\right\}=\operatorname{diag}\left[\sigma_{1}^{2}, \sigma_{2}^{2}, \ldots, \sigma_{N}^{2}\right]
$$

The following assumptions are made in the subsequent developments.

- $\quad$ The number of sources $P$ is known a priori (otherwise, can be estimated by [1]) and the number of sensors satisfies $N \geq 3 P$. Although the condition is mild in some practice applications, this is a weakness of the proposed method.

- $\quad$ The set of $P$ steering vectors is linearly independent.

- $\quad$ The $P$ signal sources are statistically independent of each other.

The problem of interest here is to estimate the noise covariance $\mathbf{Q}$ (i.e., $\left\{\sigma_{1}^{2}, \ldots, \sigma_{N}^{2}\right\}$ ) and further obtain unknown parameters $\left\{\theta_{1}, \ldots, \theta_{P}\right\}$ from the observed array output.

\section{PROPOSED METHOD}

We first define the following diagonal matrices from the $\mathbf{Q}$ :

$$
\begin{aligned}
& \mathbf{Q}_{1}=\operatorname{diag}\left[\sigma_{1}^{2}, \ldots, \sigma_{P}^{2}\right] \\
& \mathbf{Q}_{2}=\operatorname{diag}\left[\sigma_{P+1}^{2}, \ldots, \sigma_{2 P}^{2}\right] \\
& \mathbf{Q}_{3}=\operatorname{diag}\left[\sigma_{2 P+1}^{2}, \ldots, \sigma_{N}^{2}\right] .
\end{aligned}
$$

One first defines a transfer matrix $\mathbf{D}$ as follows:

$$
\mathbf{D}=\left[\mathbf{I}_{3 P \times 3 P} \mathbf{O}_{3 P \times(N-3 P)}\right]_{3 P \times N}
$$

where $\mathbf{I}_{3 P \times 3 P}$ is an identical matrix and $\mathbf{O}_{3 P \times(N-3 P)}$ a zero matrix, respectively.

To estimate $\mathbf{Q}_{1}$ and $\mathbf{Q}_{2}$, one needs to reduce the dimension of $\mathbf{R}_{\mathbf{x x}}$ using the transfer matrix D. From (2), one can get the following:

$$
\begin{aligned}
\mathbf{R}_{D} & =\mathbf{D} \mathbf{R}_{\mathbf{x x}} \mathbf{D}^{T} \\
& =\mathbf{D A} \mathbf{R}_{s s} \mathbf{A}^{H} \mathbf{D}^{T}+\mathbf{D Q D}^{T} \\
& =\mathbf{B} \mathbf{R}_{s s} \mathbf{B}^{H}+\mathbf{Q}_{D}
\end{aligned}
$$

where

$$
\begin{aligned}
& \mathbf{B}=\mathbf{D A}=\left[\begin{array}{lll}
\mathbf{B}_{1_{P \times P}^{T}}^{T} & \mathbf{B}_{2_{P \times P}}^{T} & \mathbf{B}_{3_{P \times P}}^{T}
\end{array}\right]_{3 P \times P}^{T} \\
& \mathrm{Q}_{D}=\mathrm{DQD}^{T}=\left(\begin{array}{ccc}
\mathrm{Q}_{1} & \mathbf{0} & \mathbf{0} \\
\mathbf{0} & \mathrm{Q}_{2} & \mathbf{0} \\
\mathbf{0} & \mathbf{0} & \mathrm{Q}_{33}
\end{array}\right)
\end{aligned}
$$

where all the submatrices of $\mathbf{B}$ and $\mathbf{Q}_{D}$ are $P$-order square matrices and $\mathrm{Q}_{33}$ needs not be estimated here. In the following, further partition of $\mathbf{R}_{D}$ occurs into:

$$
\mathbf{R}_{D}=\left(\begin{array}{ccc}
\mathbf{R}_{11_{P \times P}} & \mathbf{X}_{1_{P \times P}} & \mathbf{X}_{2_{P \times P}} \\
\mathbf{X}_{3_{P \times P}} & \mathbf{R}_{22_{P \times P}} & \mathbf{X}_{4_{P \times P}} \\
\mathbf{X}_{5_{P \times P}} & \mathbf{X}_{6_{P \times P}} & \mathbf{R}_{33_{P \times P}}
\end{array}\right)_{3 P \times 3 P} .
$$

All the submatrices of $\mathbf{R}_{D}$ are also $P$-order square matrices; then, we have the following:

$$
\begin{aligned}
\mathbf{R}_{11} & =\mathbf{Q}_{1}+\mathbf{B}_{1} \mathbf{R}_{s s} \mathbf{B}_{1}^{H} \quad \mathbf{R}_{22}=\mathbf{Q}_{2}+\mathbf{B}_{2} \mathbf{R}_{s s} \mathbf{B}_{2}^{H} \\
\mathbf{X}_{4} & =\mathbf{B}_{2} \mathbf{R}_{s s} \mathbf{B}_{3}^{H} \quad \mathbf{X}_{5}=\mathbf{B}_{3} \mathbf{R}_{s s} \mathbf{B}_{1}^{H} .
\end{aligned}
$$

Here $\mathbf{A}$ is of full rank according to the previous assumptions, so is $\mathbf{B}$. Therefore, both $\mathbf{X}_{4}$ and $\mathbf{X}_{5}$ are invertible matrices.

From (9), we obtain

$$
\begin{aligned}
\mathbf{Q}_{1}= & \mathbf{R}_{11}-\mathbf{B}_{1} \mathbf{R}_{s s} \mathbf{B}_{1}^{H} \\
= & \mathbf{R}_{11}-\mathbf{X}_{2} \mathbf{X}_{4}^{-1} \mathbf{X}_{3}-\mathbf{B}_{1} \mathbf{R}_{s s} \mathbf{B}_{1}^{H}+\mathbf{X}_{2} \mathbf{X}_{4}^{-1} \mathbf{X}_{3} \\
= & \mathbf{R}_{11}-\mathbf{X}_{2} \mathbf{X}_{4}^{-1} \mathbf{X}_{3}-\mathbf{B}_{1} \mathbf{R}_{s s} \mathbf{B}_{1}^{H} \\
& +\mathbf{B}_{1} \mathbf{R}_{s s} \mathbf{B}_{3}^{H} \mathbf{B}_{3}^{-1^{H}} \mathbf{R}_{s s}^{-1} \mathbf{B}_{2}^{-1} \mathbf{B}_{2} \mathbf{R}_{s s} \mathbf{B}_{1}^{H} \\
= & \mathbf{R}_{11}-\mathbf{X}_{2} \mathbf{X}_{4}^{-1} \mathbf{X}_{3} .
\end{aligned}
$$

Similarly, one has

$$
\mathbf{Q}_{2}=\mathbf{R}_{22}-\mathbf{X}_{3} \mathbf{X}_{5}^{-1} \mathbf{X}_{6}
$$

Seen from the estimations of $\mathbf{Q}_{1}$ and $\mathbf{Q}_{2}$, the number of sensors $N$ needs to satisfy $N \geq 3 P$ for the proposed method to work.

To give an estimate of $\mathrm{Q}_{3}$ and now partition the covariance matrix $\mathbf{R}_{\mathbf{x x}}$, the following occurs:

$\mathbf{R}_{\mathbf{x x}}=\left(\begin{array}{ccc}\mathbf{X X}_{P \times P} & \mathbf{X X}_{P \times P} & \mathbf{X X}_{P \times(N-2 P)} \\ \mathbf{R}_{1 P \times P} & \mathbf{X X}_{P \times P} & \mathbf{R}_{3_{P \times(N-2 P)}} \\ \mathbf{R}_{2(N-2 P) \times P} & \mathbf{X} \mathbf{X}_{(N-2 P) \times P} & \mathbf{R}_{4_{(N-2 P) \times(N-2 P)}}\end{array}\right)$ where $\mathbf{X X}$ denotes block without importance for the present discussion.

Let the row of $\mathbf{A}$ be partitioned as

$$
\mathbf{A}=\left(\begin{array}{c}
\mathbf{A}_{1 P \times P} \\
\mathbf{A}_{2 P \times P} \\
\mathbf{A}_{3(N-2 P) \times P}
\end{array}\right)_{N \times P}
$$

According to (2) and using the above partition of $\mathbf{A}$, one can obtain easily

$$
\begin{aligned}
& \mathbf{R}_{\mathbf{x x}}=\left(\begin{array}{c}
\mathbf{A}_{1} \\
\mathbf{A}_{2} \\
\mathbf{A}_{3}
\end{array}\right) \mathbf{R}_{s s}\left[\begin{array}{lll}
\mathbf{A}_{1}^{H} & \mathbf{A}_{2}^{H} & \mathbf{A}_{3}^{H}
\end{array}\right]+\mathbf{Q} \\
& =\left(\begin{array}{ccc}
\mathbf{X X} & \mathbf{X X} & \mathbf{X X} \\
\mathbf{A}_{2} \mathbf{R}_{\mathbf{s s}} \mathbf{A}_{1}^{H} & \mathbf{X X} & \mathbf{A}_{2} \mathbf{R}_{\mathbf{s s}} \mathbf{A}_{3}^{H} \\
\mathbf{A}_{3} \mathbf{R}_{\mathbf{s s}} \mathbf{A}_{1}^{H} & \mathbf{X X} & \mathbf{A}_{3} \mathbf{R}_{\mathbf{s s}} \mathbf{A}_{3}^{H}+\mathbf{Q}_{3}
\end{array}\right)
\end{aligned}
$$

and, hence, one has

$$
\begin{aligned}
& \mathbf{R}_{1}=\mathbf{A}_{2} \mathbf{R}_{s s} \mathbf{A}_{1}^{H} \\
& \mathbf{R}_{2}=\mathbf{A}_{3} \mathbf{R}_{s s} \mathbf{A}_{1}^{H} \\
& \mathbf{R}_{3}=\mathbf{A}_{2} \mathbf{R}_{s s} \mathbf{A}_{3}^{H} \\
& \mathbf{R}_{4}=\mathbf{A}_{3} \mathbf{R}_{s s} \mathbf{A}_{3}^{H}+\mathbf{Q}_{3} .
\end{aligned}
$$


Based on the previous assumptions, the following is easily derived from (13)

$$
\begin{aligned}
\mathbf{Q}_{3}= & \mathbf{R}_{4}-\mathbf{A}_{3} \mathbf{R}_{s s} \mathbf{A}_{3}^{H} \\
= & \mathbf{R}_{4}-\mathbf{R}_{2} \mathbf{R}_{1}^{-1} \mathbf{R}_{3} \\
& +\mathbf{A}_{3} \mathbf{R}_{s s} \mathbf{A}_{1}^{H} \mathbf{A}_{1}^{-H} \mathbf{R}_{s s}^{-1} \mathbf{A}_{2}^{-1} \mathbf{A}_{2} \mathbf{R}_{s s} \mathbf{A}_{3}^{H}-\mathbf{A}_{3} \mathbf{R}_{s s} \mathbf{A}_{3}^{H} \\
= & \mathbf{R}_{4}-\mathbf{R}_{2} \mathbf{R}_{1}^{-1} \mathbf{R}_{3} .
\end{aligned}
$$

Noise variance vector $\left\{\sigma_{2 P+1}^{2}, \ldots, \sigma_{N}^{2}\right\}$ can be estimated by the diagonal elements of $\mathbf{Q}_{3}$.

In real application, the array covariance matrix is estimated by the finite sample data of array

$$
\hat{\mathbf{R}}_{\mathbf{x x}}=\frac{1}{K} \sum_{t=1}^{K} \hat{\mathbf{x}}(t) \hat{\mathbf{x}}^{H}(t) .
$$

Therefore, an estimation of the noise-covariance matrix $\mathbf{Q}$ is obtained by the estimation of $\mathrm{Q}_{1}, \mathrm{Q}_{2}$, and $\mathrm{Q}_{3}$, as shown in (16) at the bottom of the page. Using the estimated noise-covariance matrix, we may prewhiten the received array data and then obtain the improvement of performance in colored noise. The computation procedure of the proposed method is summarized as follows.

- $\quad$ Compute $\hat{\mathbf{R}}_{\mathbf{x x}}=(1 / K) \sum_{t=1}^{K} \mathbf{x}(t) \mathbf{x}^{H}(t)$ and obtain the estimate of $\hat{\mathbf{Q}}$ by (16).

- Use the estimated noise-covariance matrix $\hat{\mathbf{Q}}$ to prewhiten the received data $\hat{\mathbf{x}}(t)$ and yield

$$
\hat{\mathbf{R}}_{w}=\hat{\mathbf{Q}}^{-1 / 2} \hat{\mathbf{R}}_{x x} \hat{\mathbf{Q}}^{-1 / 2} \text {. }
$$

- Obtain the noise subspace by the eigendecomposition of $\hat{\mathbf{R}}_{w}$

$$
\hat{\mathbf{E}}_{\mathbf{N}}=\left[\hat{\mathbf{e}}_{1}, \ldots, \hat{\mathbf{e}}_{N-P}\right] .
$$

- $\quad$ Compute the spatial spectrum function and obtain the estimate of $\theta_{k}(k=1,2, \ldots, P)$ from $P$ spectrum peaks

$$
f(\theta)=\frac{1}{\sum_{i=1}^{N-P}\left|\left(\hat{\mathbf{Q}}^{-1 / 2} \mathbf{a}(\theta)\right)^{H} \hat{\mathbf{e}}_{i}\right|^{2}}
$$

Regarding main computational complexity in the proposed method, the estimate of noise-covariance matrix $\hat{\mathbf{Q}}$ only requires linear operations and is computationally simpler, while the number of multiplications involved in calculating both
$\hat{\mathbf{Q}}^{-1 / 2}$ and the eigendecomposition of $\hat{\mathbf{R}}_{w}$ is in the order of $O\left(N^{3}\right)$. The computation of spatial spectrum function is in the order of $O\left(N^{2} m\right)$, where $m$ is the number of searches conducted along the angle axis. However, in ML algorithm [8], the update of the likelihood function at each iteration needs also to compute the $\hat{\mathrm{Q}}^{-1 / 2}$, while the ML method uses the uniform noise assumption to obtain the initialized values of angle estimates. The ML algorithm requires additional computations to upgrade the nuisance parameters and normalizes the data vector at each iteration. This additional complexity increases linearly as $N$ or $K$ increase. However, the major computation cost of the ML method is that a highly nonlinear (global) optimization problem should be repeatedly solved, and the computational complexity increases nonlinearly as $P$ increase. Ignoring the same computational load, therefore, the computational complexity of the proposed method is much lower than that of the ML algorithm.

\section{Simulation RESUlts}

In the first simulation, the experiments are performed with a uniform linear array (ULA) with $N=9$ sensors and half-wavelength interelement spacing. Two equally powered narrowband sources with DOAs $\theta_{1}=-4^{\circ}$ and $\theta_{2}=5^{\circ}$ impinge on the array and the two sources are statistically independent of each other. We assume the spatially nonuniform independent sensor noise has the following covariance matrix:

$$
\mathbf{Q}=\sigma_{n}^{2} \operatorname{diag}[1,1.2,4,12,11,3,0.4,10,2] .
$$

The signal-to-noise ratio (SNR) is defined as

$$
\mathrm{SNR}=10 \log 10\left(\frac{\sigma_{s}^{2}}{\frac{\sigma_{n}^{2}}{N} \sum_{i=1}^{N} \sigma_{i}^{2}}\right)
$$

where $\sigma_{s}^{2}$ denotes the power of source signal. The estimate results are obtained from $K=20$ snapshots and 20 independent runs. In Figs. 1 and 4-6, the marks for different methods are respectively given: "solid line": Proposed method; "+": Difference approach [7]; ":": MUSIC without prewhitening; “*”: Capon method; "dashed line": Conventional beamforming.

The results of five methods are compared in Fig. 1. Seen from Fig. 1, the performance of the prewhitened MUSIC method using the estimated noise covariance is improved while the MUSIC method without prewhitening, Capon method, conventional beamforming, and a transform-based covariance difference method [7] are all failed to resolve the two closely

$$
\hat{\mathbf{Q}}=\left(\begin{array}{ccc}
\operatorname{diag}\left\{\hat{\mathbf{R}}_{11}-\hat{\mathbf{X}}_{2} \hat{\mathbf{X}}_{4}^{-1} \hat{\mathbf{X}}_{3}\right\} & \mathbf{0} & \mathbf{0} \\
\mathbf{0} & \operatorname{diag}\left\{\hat{\mathbf{R}}_{22}-\hat{\mathbf{X}}_{3} \hat{\mathbf{X}}_{5}^{-1} \hat{\mathbf{X}}_{6}\right\} & \mathbf{0} \\
\mathbf{0} & \mathbf{0} & \operatorname{diag}\left\{\hat{\mathbf{R}}_{4}-\hat{\mathbf{R}}_{2} \hat{\mathbf{R}}_{1}^{-1} \hat{\mathbf{R}}_{3}\right\}
\end{array}\right)
$$




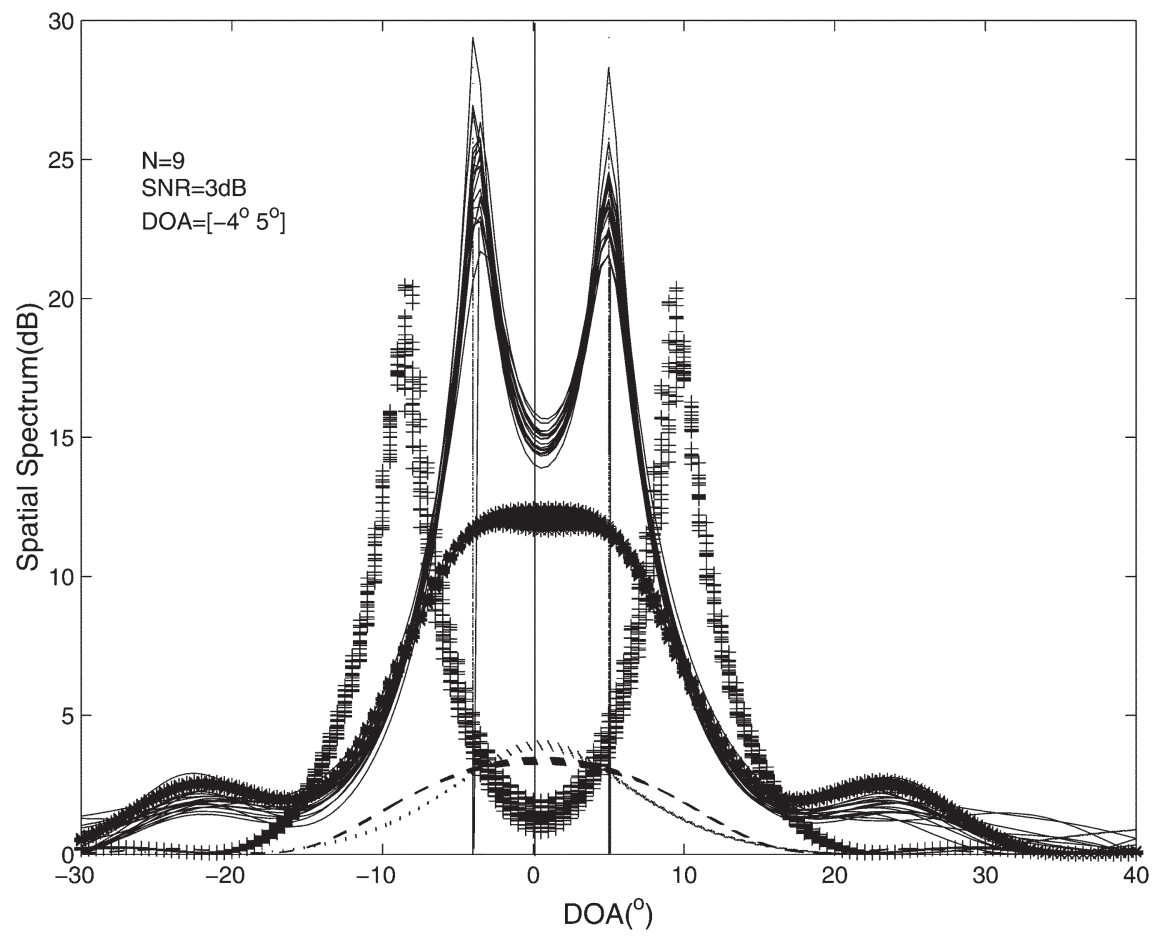

Fig. 1. Simulation results for 20 runs. The other parameters are SNR = 3 dB, 20 snapshots, and 9 sensors. "Solid line": Proposed method; "+": Difference approach [7]; ":”: MUSIC without prewhitening; “*”: Capon method; "dashed line”: Conventional beamforming.

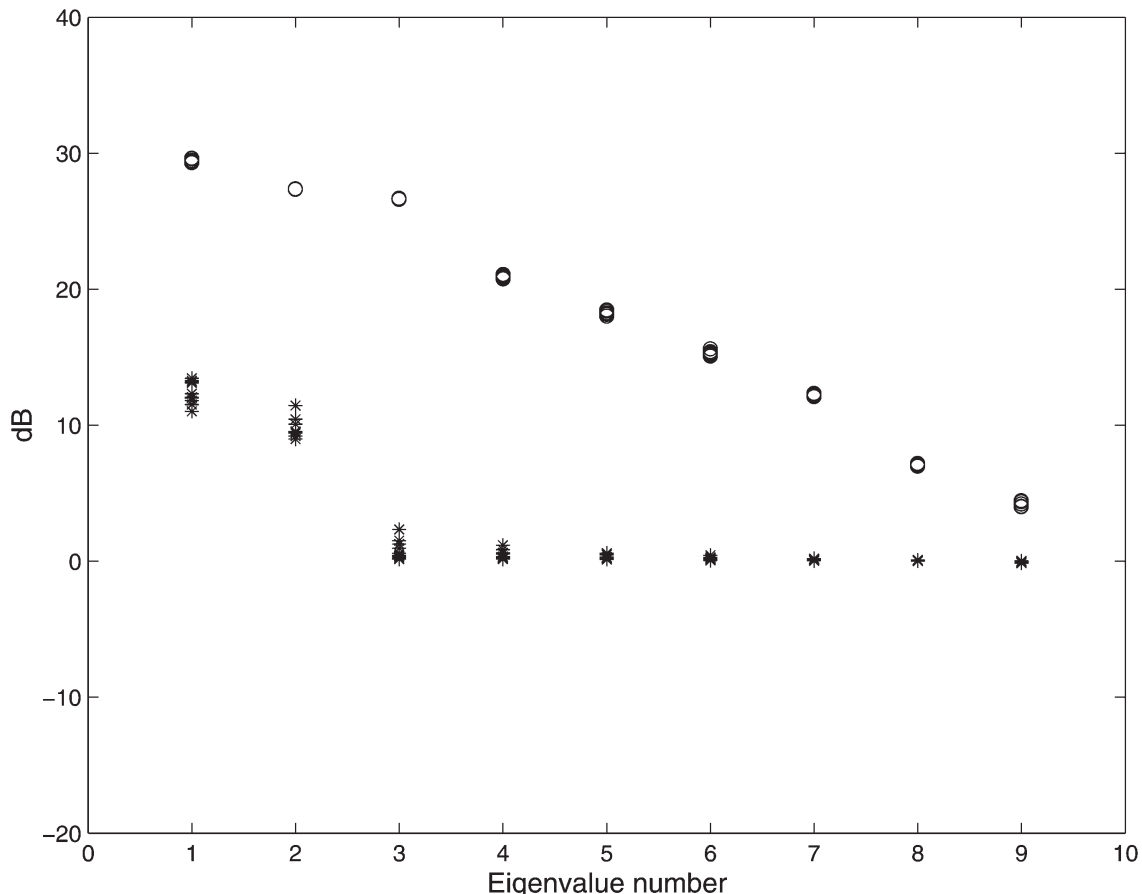

Fig. 2. Simulation results for 20 runs. The other parameters are 20 snapshots, and 9 sensors. " $*$ " denotes the eigenvalues of the proposed method; "o" denotes the eigenvalues of MUSIC method without prewhitening.

spaced signal sources. On the other hand, as shown in Fig. 2, the smallest eigenvalues of the conventional MUSIC method will be perturbed from their true values so that the signal subspace as well noise subspace cannot be accurately separated. The root-mean-square errors (RMSEs) of DOA estimation of the proposed method has been compared with those of the MUSIC without prewhitening and Capon method. Fig. 3 shows that the performances of the latter two methods become poor in lower SNR cases. The results for source 1 are similar and, hence, omitted. It is further seen from Fig. 4 that the performances of MUSIC method with prewhitening, MUSIC method without prewhitening, and Capon method are nearly identical when the 


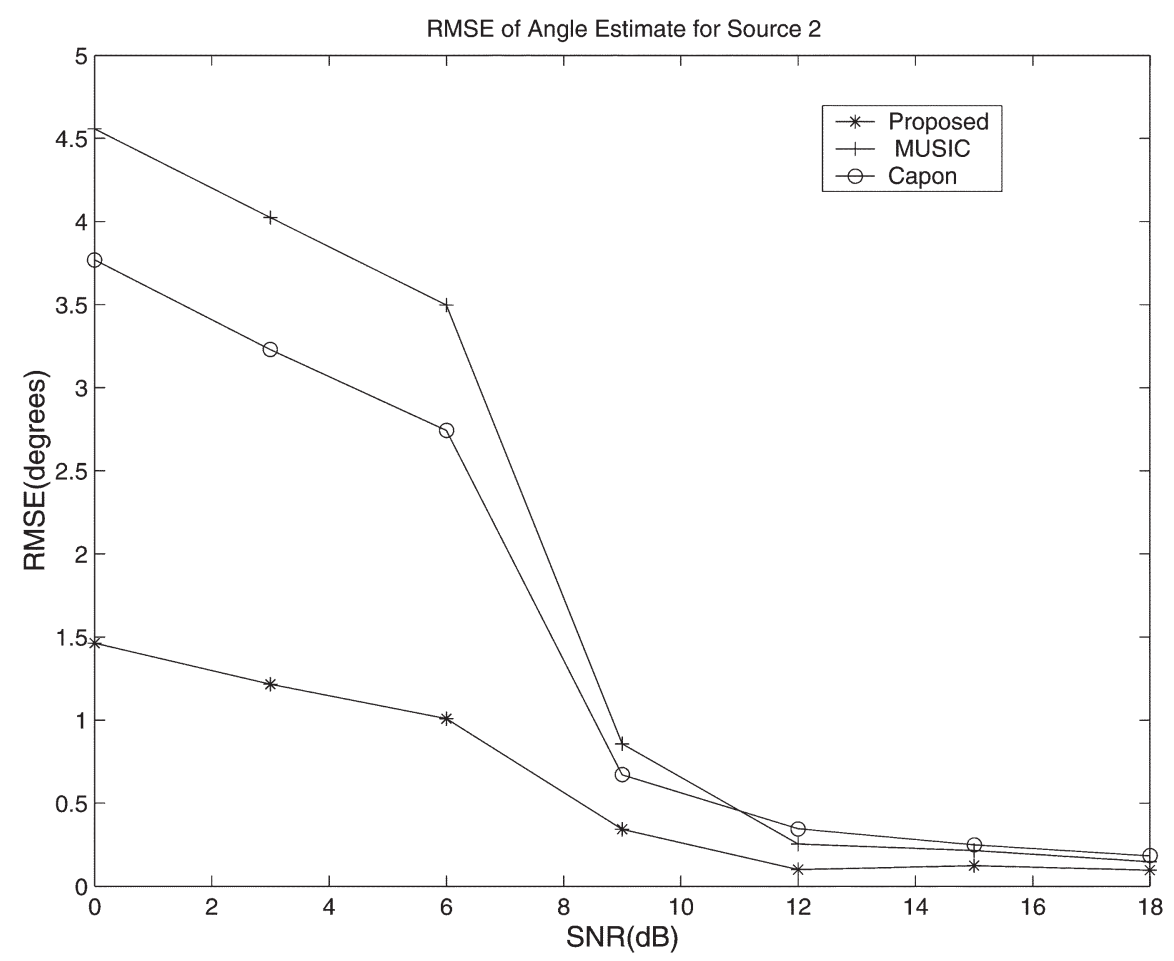

Fig. 3. Simulation results for 50 runs. The other parameters are 20 snapshots, and 9 sensors."*”: Proposed method; "+": MUSIC without prewhitening; "o": Capon method.

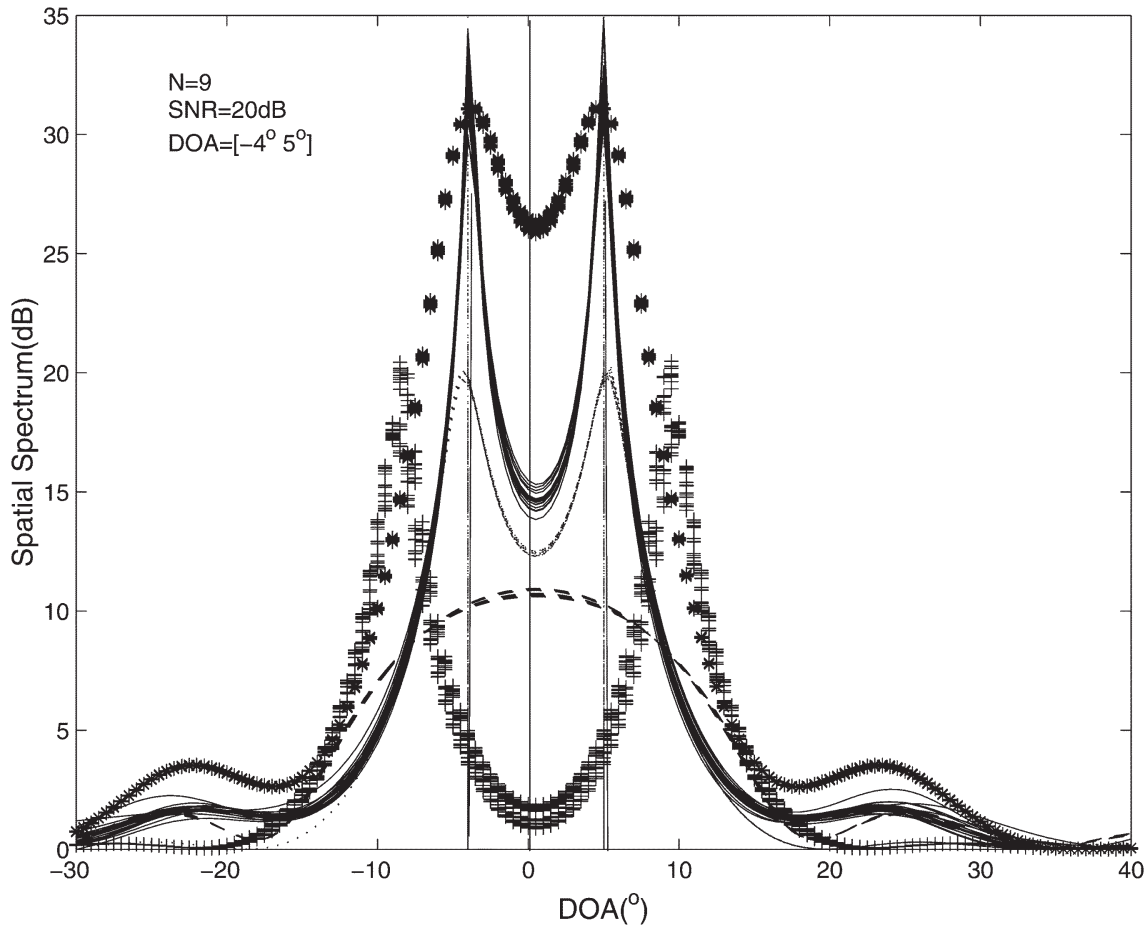

Fig. 4. Simulation results for 20 runs. The other parameters are SNR $=20 \mathrm{~dB}, 20$ snapshots, and 9 sensors. "Solid line": Proposed method; "+": Difference approach [7]; “:”: MUSIC without prewhitening; “*”: Capon method; “dashed line”: Conventional beamforming.

SNR is high enough. Both Figs. 3 and 4 also show that for a higher SNR case, the degradation due to the colored noise is negligible. In particular, the performances of the five methods are also compared in white Gaussian noise, seen from Fig. 5. The performances of the proposed method, MUSIC method, and Capon method become nearly identical.
In the second simulation, the spatially nonuniform independent sensor noise covariance has the following matrix:

$$
\mathbf{Q}=\sigma_{n}^{2} \operatorname{diag}[1,1.2,4,5,7,3] .
$$

The number of sensors is $N=6$ (i.e., small number of sensors) and the $\mathrm{SNR}=5 \mathrm{~dB}$ is taken. As expected in Fig. 6, the 


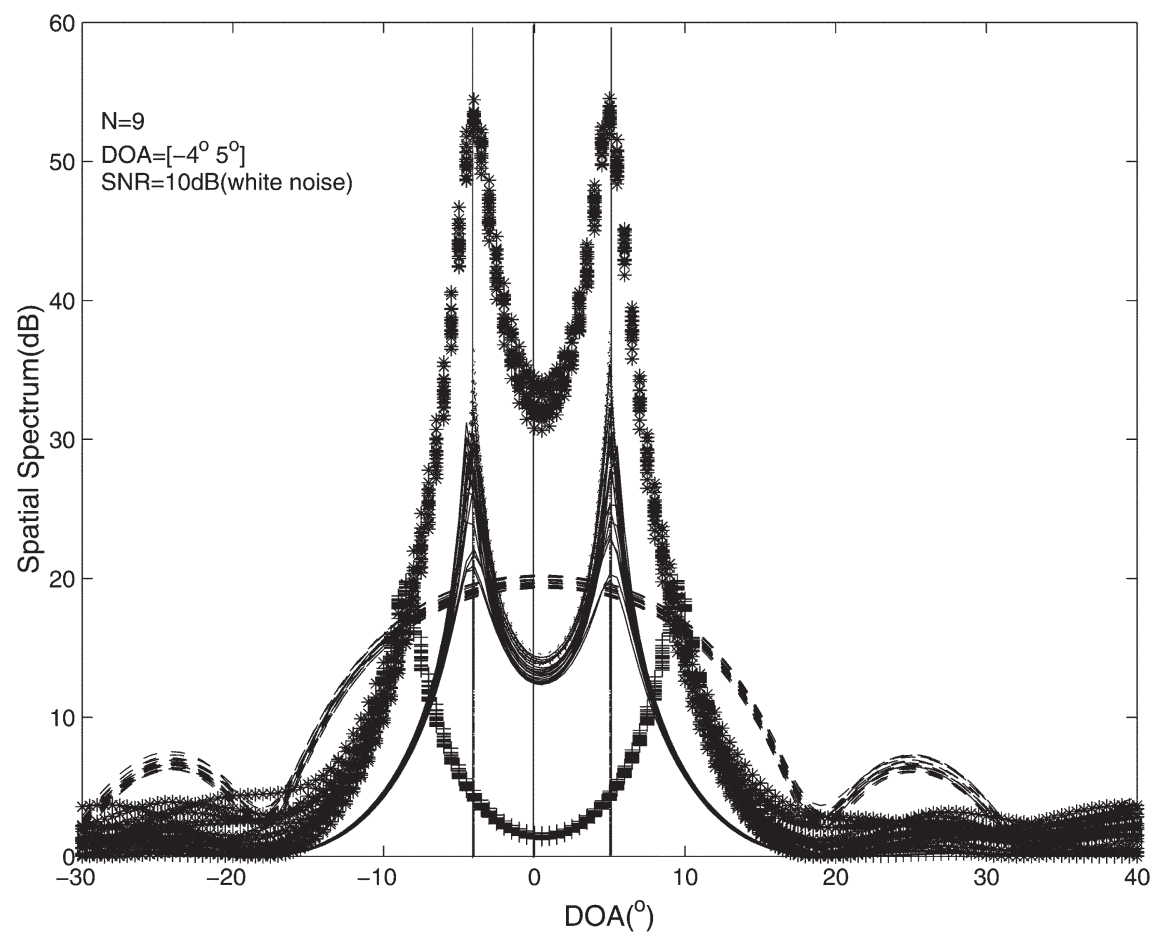

Fig. 5. Simulation results for 20 runs in white noise. The other parameters are SNR $=10 \mathrm{~dB}, 50$ snapshots, and 9 sensors. "Solid line": Proposed method; " + ": Difference approach [7]; ":”: MUSIC without prewhitening; “*”: Capon method; "dashed line”: Conventional beamforming.

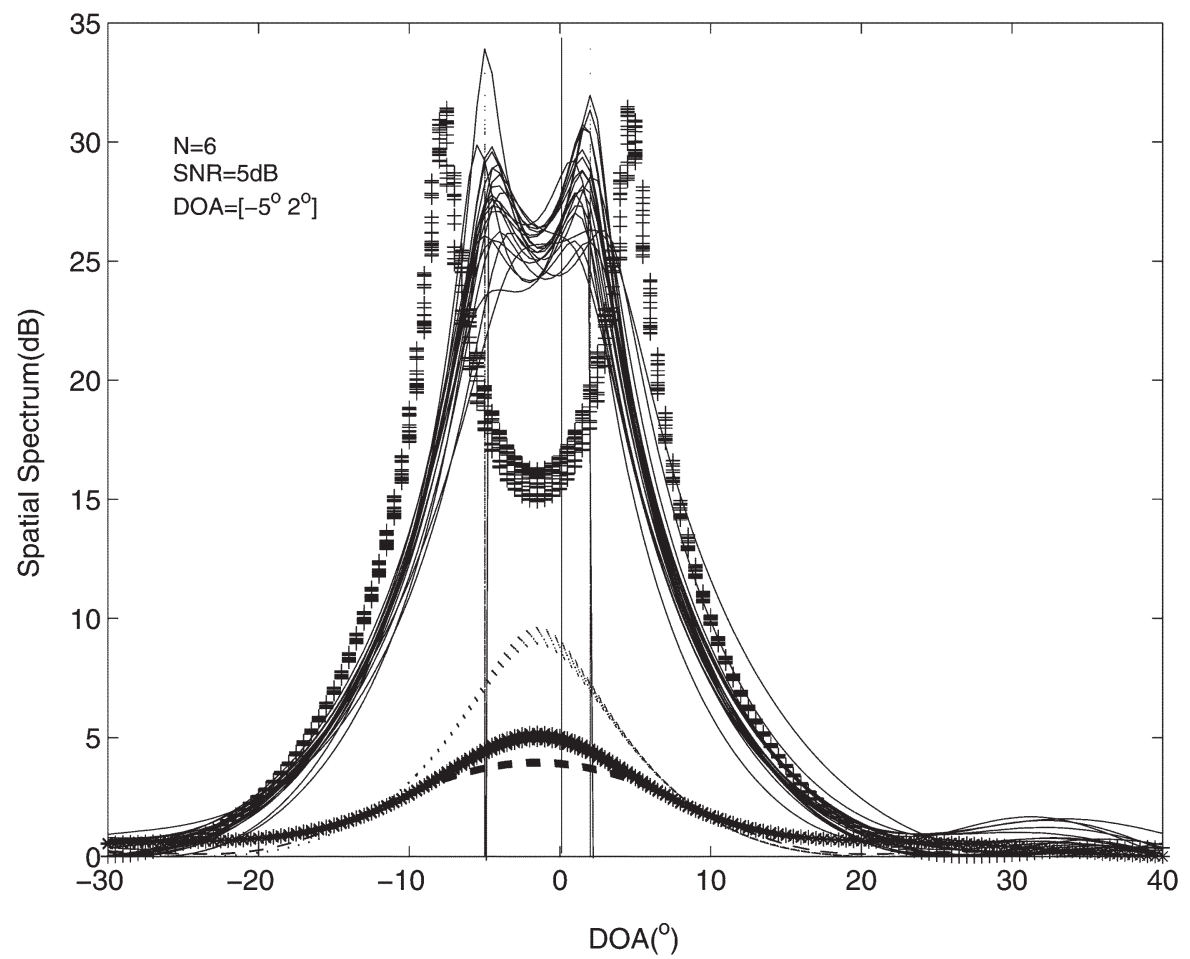

Fig. 6. Simulation results for 20 runs. The other parameters are SNR $=5$ dB, 20 snapshots, and 6 sensors. "Solid line": Proposed method; "+": Difference approach [7]; “:”: MUSIC without prewhitening; “*”: Capon method; "dashed line”: Conventional beamforming.

proposed method with prewhitening provides essential performance improvements relative to the rest four methods when the number of sensors is small.

\section{CONCLUSION}

The problem of estimation the DOAs of multiple sources in the presence of spatial nonuniform independent sensor noises is considered. A new estimator of noise-covariance matrix based on the developed nonuniform noise model is derived and, hence, the received data of sensor array can be directly prewhitened using the estimated noise-covariance matrix. Finally, simulation results show the performance improvements of the standard MUSIC method using the modified array data achieved. A weakness of the proposed method is that the number of sensors 
$N$ needs to satisfy $N \geq 3 P$, while the proposed method does not consider the effect of array manifold error. One needs to further improve our method in the future work.

\section{ACKNOWLEDGMENT}

The authors would like to thank the Associate Editor and the anonymous reviewers for their valuable comments and suggestions on the original manuscript.

\section{REFERENCES}

[1] Yuehua, Wu, and K.-W. Tam, "On determination of the number of signals in spatially correlated noise," IEEE Trans. Signal Process., vol. 44, no. 11 , pp. 3023-3029, Nov. 1998.

[2] H. Sinath and V. U. Reddy, "Analysis of MUSIC algorithm with sensor gain and phase preturbations," Signal Process., vol. 23, no. 3, pp. 245-256, 1991.
[3] Li Fu and R. Vaccaro, "Performance degradation of DOA estimators due to unknown noise fields," IEEE Trans. Signal Process., vol. 40, no. 3, pp. 686-690, Mar. 1992.

[4] B. Goransson and B. Ottersten, "Direction estimation in partially unknown noise fields," IEEE Trans. Signal Process., vol. 47, no. 9, pp. 2375-2384, Sep. 1999.

[5] M. Agrawal and S. Prasad, "A modified likelihood function appraoch to DOA estimation in the presence of unknown spatially correlated Gaussian noise using a uniform linear array," IEEE Trans. Signal Process., vol. 48, no. 10, pp. 2743-2749, Oct. 2000.

[6] A. Paulraj and T. Kailatn, "Eigenstructure method for direction of arrival estimation in the presence of unknown noise fields," IEEE Trans. Acoust., Speech, Signal Process., vol. 34, no. 1, pp. 13-20, Feb. 1986.

[7] A. Moghaddamjoo, "Transform-based covariance difference approach to the array with spatially nonstationary noise," IEEE Trans. Signal Process., vol. 39, no. 1, pp. 219-221, Jan. 1991.

[8] M. Pesavento and A. B. Gershman, "Maximum-likelihood direction-ofarrival estimation in the presence of unknown nonuniform noise," IEEE Trans. Signal Process., vol. 49, no. 7, pp. 1310-1324, Jul. 2001.

[9] S. A. Vorobyov, A. B. Gershman, and K. M. Wong, "Maximum likelihood Direction-of-Arrival estimation in unknown noise fields using sparse sensor arrays," IEEE Trans. Signal Process., vol. 53, no. 1, pp. 34-43, Jan. 2005

[10] P. Stoica and T. Soderatrom, "On estimating the noise power in array processing," Signal Process., vol. 26, no. 2, pp. 205-220, 1992. 